\title{
Nasobiliary Drain Diverted through a Percutaneous Endoscopic Gastrostomy Tube: A Novel Approach to Nasobiliary Drainage
}

\author{
Nikolaos Dimitrios Pantzaris ${ }^{a}$ Tim Lord $^{b}$ Robyn Sotheran ${ }^{c}$ \\ John Hutchinson ${ }^{a}$ Charles Millson ${ }^{a}$ \\ aHepatology Department, York Teaching Hospital NHS Foundation Trust, York, UK; \\ bEndoscopy Department, Scarborough Hospital, Scarborough, UK; ' ${ }^{\mathrm{b}}$ Medical Illustration \\ Department, York Teaching Hospital NHS Foundation Trust, York, UK
}

\author{
Keywords \\ Primary biliary cholangitis · Pruritus · Nasobiliary drain · Biliary drain · Percutaneous \\ endoscopic gastrostomy
}

\begin{abstract}
Intractable pruritus is a common, debilitating symptom and a well-defined entity occurring in chronic cholestatic disorders. Treatment options include cholestyramine, rifampicin, naItrexone, gabapentin, and sertraline, as well as more interventional measures, such as plasmapheresis, extracorporeal albumin dialysis, nasobiliary drains (NBDs), and UVB phototherapy in patients who fail to respond to medical therapy. Despite the limited data, NBD seems to be a highly effective treatment in the relief of refractory cholestatic pruritus. In this article, we present the case of a 73-year-old woman with primary biliary cholangitis and intractable pruritus, refractory to medical treatment. The patient had a complete resolution of her symptoms following an NBD placement, in which, with a novel approach, the nasal end was redirected and exited through a percutaneous endoscopic gastrostomy port, significantly improving her quality of life.
\end{abstract}




\section{Introduction}

Pruritus is a very common and often debilitating symptom of chronic, cholestatic liver disorders. In primary biliary cholangitis (PBC) patients, pruritus may be the sole presenting symptom, although severity frequently correlates poorly with liver function tests or disease duration [1]. Whilst the pathogenesis of pruritus in cholestasis remains unclear, bile salts, endogenous opioids, serotonin, histamine, and progesterone metabolites have all been postulated as potential pruritogens. However, no causative link or association with intensity has been established for any of these molecules. Recently, lysophosphatidic acid and its forming enzyme, autotaxin, have also been reported as having a key role in the pathophysiology of cholestatic itch $[2,3]$.

The treatment options for pruritus include bile acid resins (cholestyramine or colesevelam), rifampicin, opioid antagonists naltrexone or naloxone, gabapentin, and the serotonin re-uptake inhibitor, sertraline. These medications are used in a stepwise therapeutic approach; however, none have a very strong evidence base, and efficacy is very variable indeed. For those patients who fail to respond to medical therapy, more interventional measures, such as plasmapheresis, extracorporeal albumin dialysis, and nasobiliary drains (NBDs), or experimental therapies such as UVB phototherapy [4] are available. Finally, the option of liver transplantation should be discussed, but in reality, if pruritus is the sole indication, patients can expect a long wait, whilst others with established liver failure inevitably take priority.

Although data are limited, NBD seems to be highly effective in the relief of cholestatic pruritus, but the duration of successful treatment depends on patient tolerance of the catheter (especially the nasal part) [5] and technical issues such as catheter displacement. We present the case of a 73-year-old woman with PBC and intractable pruritus refractory to medical treatment, whose symptoms resolved completely following an NBD placement. Patient tolerance was significantly improved and catheter displacement controlled by re-directing the nasal end of the NBD out through a percutaneous endoscopic gastrostomy (PEG) port.

\section{Case Report}

This 73-year-old patient presented initially in 2008 to her family doctor with generalised pruritus. Initial investigations revealed an ALT 121 IU/L (10-36) and ALP 571 IU/L (32-104). In clinic, she was noted to be slim, on no medication, drunk minimal alcohol, and otherwise enjoyed good health. Further investigations included a negative anti-mitochondrial antibody (AMA), a positive smooth muscle antibody, and anti-nuclear antibody. The immunoglobulin profile was unremarkable, and the remainder of the liver screen was negative for all other liver conditions (negative: hepatitis B, hepatitis C, ANCA, and alpha 1 antitrypsin level), and an ultrasound scan was normal. A liver biopsy was performed, which revealed some subtle inflammatory changes only. The patient was commenced on ursodeoxycholic acid at $13 \mathrm{mg} / \mathrm{kg}$ for presumed AMA-negative PBC, and her liver function improved (ALT 43 IU/L and ALP 251 IU/L).

In 2013, the patient re-presented complaining of worsening pruritus and 3-kg weight loss. She did not suffer from fatigue or other PBC-associated symptoms; indeed she remained well and active otherwise, with a WHO performance score of 0 . The patient reported an alcohol intake of 1-2 units weekly and remained on the same dose of ursodeoxycholic acid, with occasional antihistamines only. Her BMI was $21 \mathrm{~kg} / \mathrm{m}^{2}$, she had some marks on her abdomen due to pruritus, and her liver edge was palpable. The ALP was 309 IU/L (32-132) and ALT 61 IU/L (10-36). Once again, the AMA was negative, whilst the smooth muscle antibody and anti-nuclear antibody were positive with a nucleolar staining pattern.

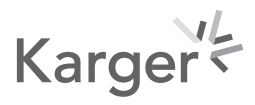


Fig. 1. High-power photomicrograph of the liver tissue, demonstrating an inflamed portal tract, an absent bile duct (ductopenia) and ductular reaction $(\times 20)$.

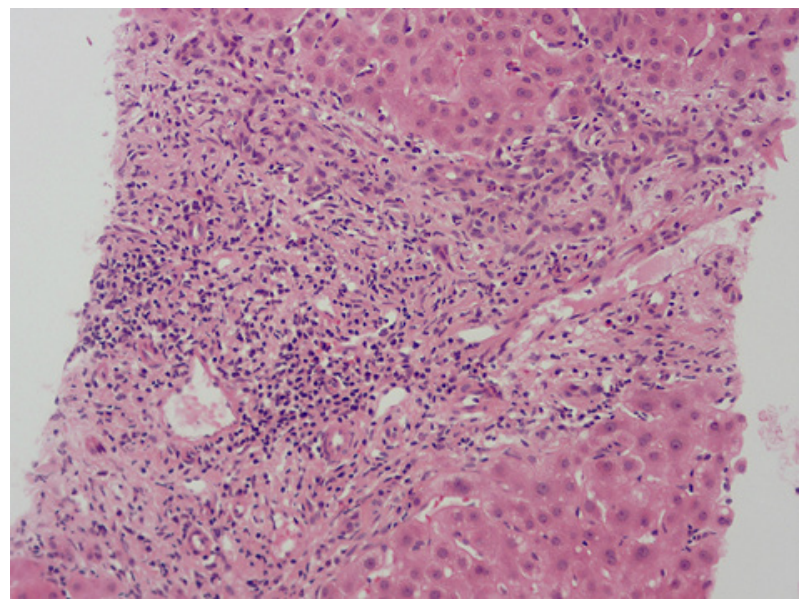

A repeat biopsy showed ductopaenia, loss of interlobular bile ducts, increase in inflammatory cells, irregular ductular proliferation, and the presence of interface hepatitis, all findings suggestive of the progressive phase of PBC (see Fig. 1). The possibility of an autoimmune hepatitis with PBC overlap syndrome was also raised by this second biopsy, but a trial of steroids did not improve the biochemistry or indeed the pruritus.

Over the next 5 years, pruritus proved to be increasingly debilitating, affecting her sleep and, as a consequence, her daytime activity. The self-scored severity score was $10 / 10$, and in the liver clinic, she was noted to have scratch marks and blood spots on her clothes and reported having to wash bed sheets after most nights' sleep. Treatments trialled for this patient's pruritus included emollient creams, cholestyramine, gabapentin, naltrexone, rifampicin, sertraline, and bezafibrate. None of these were consistently effective alone or in combination, even if there was an initial benefit. UDCA appeared to worsen the pruritus, with limited biochemical improvement, and a trial of obeticholic acid also significantly worsened her pruritus.

In 2017, our patient was referred for consideration of liver transplantation as she had tried all the antipruritic agents and continued to suffer. Bilirubin had risen to $49 \mathrm{umol} / \mathrm{L}$ (0-21), ALT 86 IU/L (0-34), ALP 343 IU/L (30-130), and albumin 38 g/L (35-50). She was deemed unsuitable because of too well-preserved liver function, but as her symptoms were not improving and her quality of life was deteriorating further, the transplant centre initiated a trial of plasma exchange. The response was striking, but the effect was limited to a maximum of 3 days after each session and then the intolerable itch returned.

Finally, in 2019, an NBD was placed as our patient approached "the end of her tether." Like plasma exchange, the NBD had an immediate, dramatic effect resolving the pruritus completely. However, after 4 weeks, the initial drain blocked, and the pruritus returned. A second NBD was placed with repeated benefit, but the patient reported that the nasal end of the drain was at times uncomfortable, and she was somewhat self-conscious about her appearance. After further discussion with the patient, it was clear that the drain was "lifechanging" in terms of the pruritus but caused daily anxiety over issues such as accidental removal and hair washing/brushing. The possibility of a biliary drain placed through a PEG tube was discussed carefully with the patient who then agreed to the procedure. See Figures 2 and 3 for the technique which was adopted.

The initial percutaneous endoscopic gastrostomy tube placement was followed by the placement of an NBD. The NBD was diverted out through the PEG -Jej extension port and attached to a collection bag. Once again, there was complete resolution of pruritus, but with no unsightly, uncomfortable, and delicate nasal drain. During the first 6 months, the drain has 


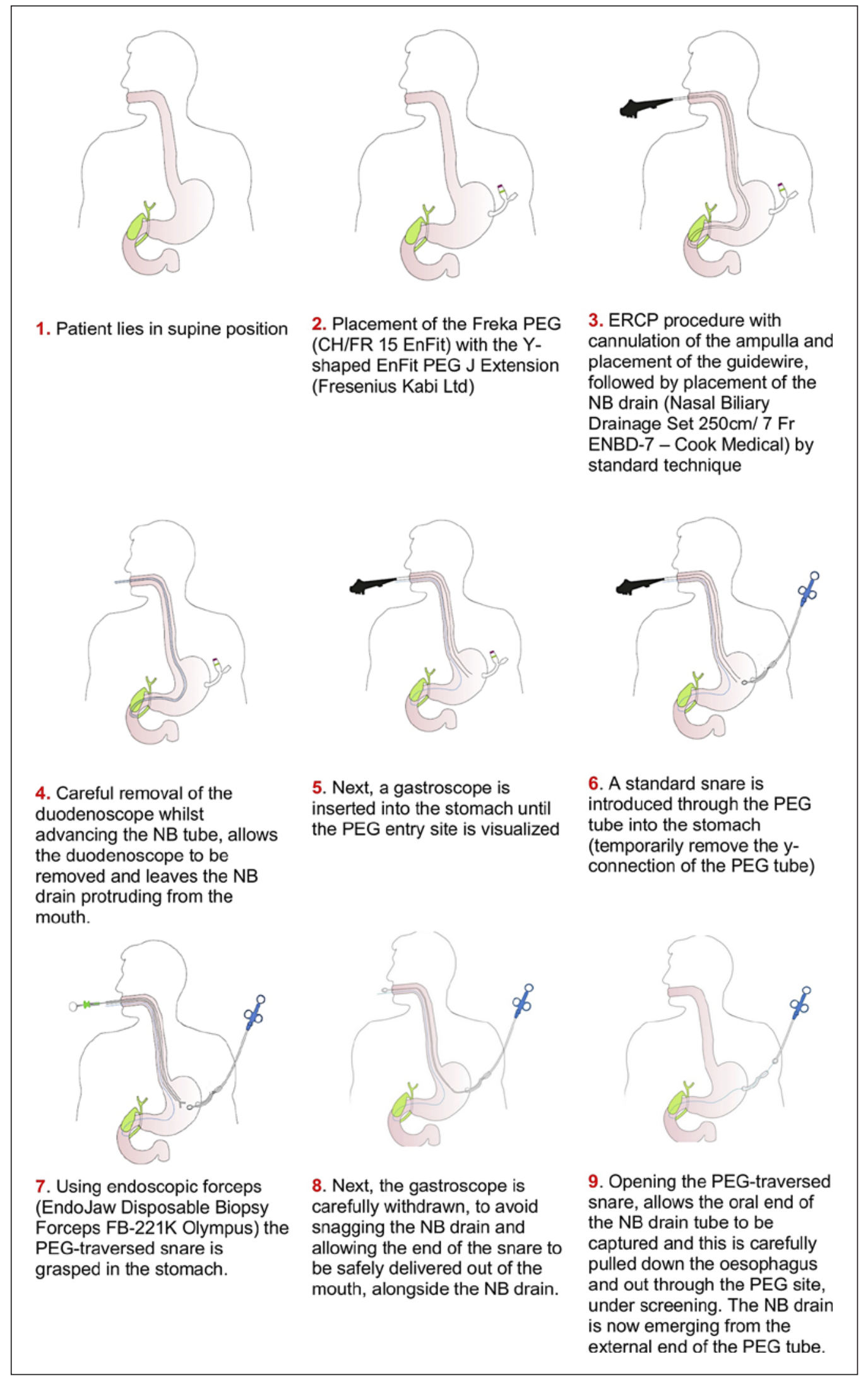

Fig. 2. PEG biliary drain placement steps 1-9. PEG, percutaneous endoscopic gastrostomy. 


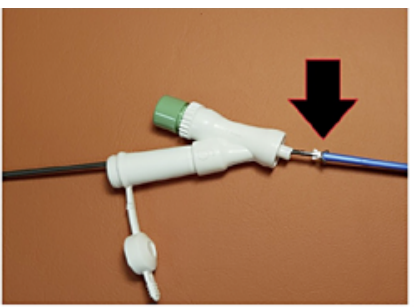

10. The attachment of the NB drain starts by passing a pair of small grasping forceps (Olympus EndoJaw Model no. FB-211K) down the flexible port of the $y$ - shaped EnFit PEG J Extension (black arrow)

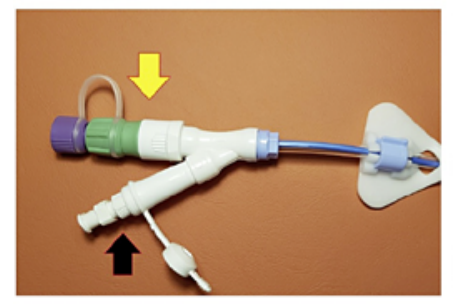

13. The NB drain locking device is pushed into the flexible PEG $\mathrm{J}$ Extension port to form a seal (black arrow). The feeding port of the EnFit PEG J Extension tube is fitted and locked in the usual way (yellow arrow).

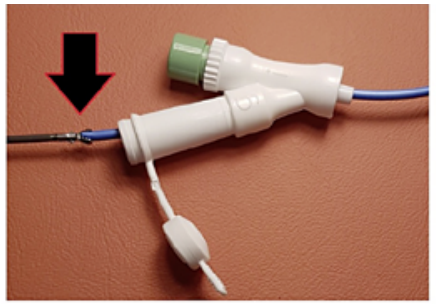

11. Then, the NB drain tube is pulled through the EnFit PEG J Extension and emerges from the flexible port (black arrow).

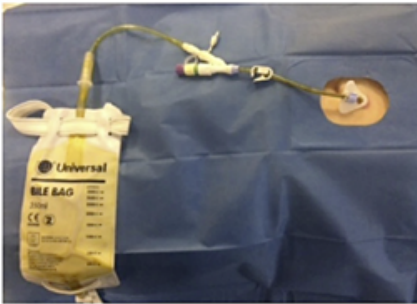

14. The final appearance shows the Bile Bag $(500 \mathrm{ml}$ Ryles/Kehrs Fitting UN405RK Universal) attached to the NB drain locking device in the standard way (this can be strapped to the patient's leg.

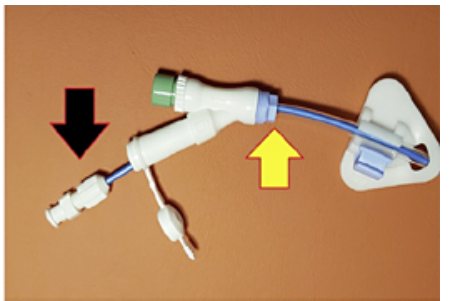

12. Next, the locking device for NB drain tube is attached in the standard way (black arrow). The other end of the EnFit PEG $\mathrm{J}$ Extension is attached to the PEG gastrostomy tubing in the usual way (yellow arrow)

Fig. 3. PEG biliary drain placement steps 10-14. PEG, percutaneous endoscopic gastrostomy.

been replaced twice, but her quality of life has been dramatically improved. The second drain placement gave 16 months of problem-free symptom relief, before it was electively exchanged. Regular checks of fat-soluble vitamins identified a vitamin A deficiency after 12 months of NBD placement. This responded to vitamin A supplementation.

\section{Discussion}

This is the first case report of a PEG-diverted nasobiliary drain to successfully treat cholestasis-associated pruritus. Data on the use of nasobiliary drainage for the management of cholestatic pruritus are sparse, but current evidence suggests that it can be a highly effective measure [5-9]. The "invasive" aspect of ERCP and NBD placement, with the attendant potential for complications, clearly deters many from even considering the option. But even if symptom relief can be assured, patient intolerance of a nasal tube, coupled with the risk of drain 
displacement, has consigned this technique largely to those patients who have failed medical treatment, herbal remedies, and experimental therapies.

The first reports of effective treatment for cholestasis related pruritus by diverting bile, date back to 1933 [10]. Since then, several studies have demonstrated that surgical external biliary drainage successfully relieves pruritus in almost all of such patients [6]. Compared to surgical drainage though, NBD is less invasive, more convenient, temporary and can be repeatedly employed. Stapelbroek et al. [7] in 2005, reported the resolution of pruritus in cholestatic BRIC patients after endoscopic NBD placement, a result that was enduring, suggesting that it should be considered as a treatment option for other cholestatic pruritus. In 2006, Beuers etal. [8] reported 3 patients with PBC with intractable pruritus who underwent NBD placement. Pruritus completely resolved within 24 hours, in 2 out of the 3 patients, lasting for 2 weeks and 1 week, respectively. The third patient reported major improvement, but only tolerated the NBD tube for 1 night [8].

A multicentre retrospective study of NBD in 2015 investigated the potential benefits and the optimal utility of NBD in intractable pruritus, of differing aetiologies. Of the 27 patients finally included in the study, all but 1 reported significant improvement in their pruritus. The median duration of benefit was 50 days, and $\mathrm{PBC}$ patients enjoyed a median of 13 further days itch-free following removal of the NBD. Adverse events occurred in 10 procedures, with 9 of them being mild post-ERCP pancreatitis. The study did not report patient preferences or technical issues such as drain displacement [5].

The only case series published regarding long-term NBD in the treatment of refractory pruritus included 2 PBC patients and 1 BRIC patient and demonstrated that this treatment modality can lead to rapid symptom relief, with no significant side effects or complications. In the first PBC patient, the NBD lasted over 12 months and in the second for over 14 months, with 1 and 3 changes of the tube, respectively, all due to blockage. Both patients experienced complete resolution of their pruritus [9]. We report here a novel approach to biliary diversion, by redirecting the nasal end of a standard NBD through a PEG port, resulting in the complete resolution of the symptoms, combined with the significant improvement on quality of life, and an easier management, without the uncomfortable and hard to tolerate nasal part of the process.

\section{Acknowledgments}

We are very grateful to Dr. Izzat Abdul-Kadir, a consultant pathologist, in York Hospital for his valuable help in the pathology slide preparation.

\section{Statement of Ethics}

Written informed consent was obtained from the patient for publication of this case report and the accompanying images. This was not an experimental study, and following advice from the research advisor in the York Teaching Hospital Foundation Trust, approval from the Health Research Authority was not necessary as it does not fall under the required categories. https://www.hra.nhs.uk/approvals-amendments/what-approvals-do-i-need/

\section{Conflict of Interests Statement}

The authors of this manuscript do not have any conflict of interest to declare.

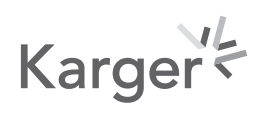




\section{Funding Sources}

This study did not receive any funding.

\section{Author Contributions}

N.D.P. reviewed the literature, and designed and wrote the manuscript. T.L. assisted with the procedure and captured the photographs. R.S. designed the illustrations. J.H. supervised and edited the manuscript. C.M. supervised and wrote the manuscript, developed the technique, and performed the procedure.

\section{References}

1 Hegade VS, Bolier R, Oude Elferink RP, Beuers U, Kendrick S, Jones DE. A systematic approach to the management of cholestatic pruritus in primary biliary cirrhosis. Frontline Gastroenterol. 2016;7:158-66.

2 Kremer AE, Namer B, Bolier R, Fischer MJ, Oude Elferink RP, Beuers U. Pathogenesis and management of pruritus in PBC and PSC. Dig Dis. 2015;33 Suppl 2:164-75.

3 Bergasa NV. The pruritus of cholestasis: facts. Hepatology. 2015;61(6):2114.

4 Mittal A. Cholestatic itch management. Curr Probl Dermatol. 2016;50:142-8.

5 Hegade VS, Krawczyk M, Kremer AE, Kuczka J, Gaouar F, Kuiper EM, et al. The safety and efficacy of nasobiliary drainage in the treatment of refractory cholestatic pruritus: a multicentre European study. Aliment Pharmacol Ther. 2016;43:294-302.

6 Hofmann AF, Huet PM. Nasobiliary drainage for cholestatic pruritus. Hepatology. 2006;43:1170-1.

7 Stapelbroek JM, van Erpecum KJ, Klomp LW, Venneman NG, Schwartz TP, van Berge Henegouwen GP, et al. Nasobiliary drainage induces long-lasting remission in benign recurrent intrahepatic cholestasis. Hepatology. 2006; 43:51-3.

8 Beuers U, Gerken G, Pusl T. Biliary drainage transiently relieves intractable pruritus in primary biliary cirrhosis. Hepatology. 2006;44:280-1.

9 Appleby VJ, Hutchinson JM, Davies MH. Safety and efficacy of long-term nasobiliary drainage to treat intractable pruritus in cholestatic liver disease. Frontline Gastroenterol. 2015;6:252-4.

10 Mocquot P. L'intervention chirurgicale dans certains icteres par retention sans obstacle. Influence du drainage biliare externe sur les functions hepatiques. J Chir. 1933;41:178-93. 